\title{
HB\&L System: rapid determination of antibiotic sensitivity of bacteria isolated from blood cultures.
}

\author{
Simone Barocci, Marinella Giacomini, Antonella Renzi, Marcello Palma, Luciano Latini, Luciano Quagliarini, \\ Antonio Migali \\ ASUR MARCHE z.t. 4 Senigallia, U.O. Patologia Clinica
}

Key words: Antimicrobial Susceptibility Test, blood culture, EUCAST.

Determinazione rapida della sensibilità agli antibiotici di batteri isolati da emocolture con sistema HB\&L

\section{SUMMARY}

Introduction. Blood culture is an important method to detect microbial pathogens on blood, very useful for diagnosing bacterial infections. Unfortunately, classical diagnostic protocols cannot directly identify bacteria responsible for sepsis and accordingly their antimicrobial profiles. This problem causes a delay of almost two days in the availability of a specific antimicrobial profile.

Objective. Among the main causes of death, sepsis have a relevant importance. For this reason it is important both to identify pathogens and to perform an antimicrobial susceptibility test in the shortest time as possible. For this purpose, the main aim of this study is the evaluation of the performances of an antimicrobial susceptibility determination directly performed on positive blood cultures.

Materials and methods. This study has been performed on 70 positive blood cultures, during the period from January to July 2009. A number of 35 blood cultures were positive for Gram negative bacteria, and 35 were positive for Gram positive bacteria. From these positive blood cultures, after a short sample preparation, it has been possible to directly determine antimicrobial susceptibility profiles by using the HB\&L (formerly URO-QUICK) instrument.

Results. The HB\&L system results showed a very good correlation with both the classical disk diffusion method and VITEK 2 automatic system. The performances between the methods carried out in this study were equivalent.

Conclusions. From data reported, thanks to the rapidity and simplicity of the method used, we can assert that the direct susceptibility test available with the HB\&L system, is useful for a rapid and early choice of the antibiotic treatment.

\section{RIASSUNTO}

Introduzione. L'emocoltura è un importante mezzo di indagine per rilevare microrganismi patogeni presenti nel sangue e risulta di grande utilità diagnostica. Purtroppo i classici protocolli diagnostici non permettono l'identificazione diretta dal flacone positivo del batterio responsabile della sepsi e, di conseguenza, l'antibiogramma dello stesso. Ciò causa un ritardo di almeno due giorni nell'approccio alla terapia.

Obiettivi. Le setticemie sono tra le principali cause di morte, per questo è importante sia identificare, che fornire un antibiogramma nel più breve tempo possibile. A tale scopo, il presente lavoro aveva come principale obiettivo la determinazione della sensibilità agli antibiotici direttamente da emocolture riscontrate positive.

Materiali e Metodi. Lo studio è stato condotto su emocolture risultate positive nel periodo da gennaio a luglio 2009, per un numero totale di 70 campioni analizzati, dei quali 35 positivi per batteri Gram negativi e 35 a Gram positivi. Dai flaconi delle emocolture positive, previa rapida preparazione dei campioni, si è proceduto mediante l'utilizzo dello strumento HB\&L all'esecuzione diretta dell'antibiogramma. Risultati. Il sistema utilizzato ha dimostrato un'ottima correlazione e prestazioni equivalenti rispetto al classico metodo di diffusione su piastra ed al sistema automatizzato VITEK 2,

Conclusioni. Da una lettura attenta dei dati, per la rapidità e la semplicità del metodo, si può affermare che l'utilizzo del sistema HB\&L per l'esecuzione di un antibiogramma diretto risulta utile per la scelta mirata e precoce dell'antibiotico da utilizzare a scopo terapeutico.

\section{INTRODUZIONE}

L'emocoltura è un importante mezzo di indagine per rilevare microrganismi patogeni presenti nel sangue e risulta di grande utilità diagnostica, in quanto permette di coltivare in arricchimento patogeni disseminati nel torrente circolatorio (BSOP37, Investigation of blood cultures for organisms other than Mycobacterium species,
Health Protection Agency, http://www.hpa-standardmethods.org.uk/documents/bsop/pdf/bsop37.pdf). Normalmente, però, dalla positività riscontrata all'emocoltura, passano almeno altri due giorni prima di ottenere uno specifico antibiogramma del batterio isolato: dal flacone positivo vengono, infatti, seminate le piastre per la crescita e successiva identificazione dell'agente di interesse e occorre poi attendere un altro giorno per avere il risultato dell'antibiogramma.

Questo fatto crea non pochi problemi legati alla necessità di sottoporre urgentemente i pazienti ad una terapia antibiotica mirata.

Esistono numerose metodiche, manuali ed automatizzate, per la determinazione della sensibilità/resistenza agli antibiotici per le varie categorie di batteri. Ognuna di queste metodiche ha sia vantaggi che svantaggi (3).

Sono molti gli studi e le comparazioni effettuati su vari sistemi per la determinazione dell'antibiotico-resistenza $(1,6,10,4,5,7)$.

L'HB\&L (ALIFAX, Italia) è stato precedentemente utilizzato per eseguire gli antibiogrammi direttamente da campioni di urine riscontrate positive ad infezioni batteriche $(8,9)$. Tale strumento risulta molto versatile per varie tipologie di matrici (2).

In considerazione di quanto esposto, abbiamo quindi pensato di utilizzarlo per eseguire l'antibiogramma direttamente sul flacone e si è proceduto nella comparazione dei dati ottenuti dal sistema HB\&L rispetto ad un metodo classico (diffusione su piastra) e ad un metodo automatizzato (VITEK 2, bioMérieux, Francia), allo scopo di fornire al clinico una risposta in tempi più celeri con l'indicazione degli antibiotici adatti da utilizzare per il paziente interessato.

\section{MATERIALI E METODI}

\section{Emocolture}

Le emocolture utilizzate nel presente lavoro sono state eseguite in un BACTECTM 9120 Blood Culture System (BD, Franklin Lakes, USA). Il sistema per emocolture Bactec è un'apparecchiatura completamente automatica per l'incuba-

\section{Corresponding author: Antonio Migali}

ASUR MARCHE z.t. 4 Senigallia - U.O. Patologia Clinica

60019 Senigallia (AN) - Via Cellini, I3 - Tel. 07I-7909 2435 - Fax 07I-7909 2439

E-mail: antonio.migali@sanita.marche.it 
zione e la rilevazione di crescita microbica in campioni di sangue.

Il campione di sangue inoculato in appositi flaconi, viene inserito all'interno dello strumento. Il sistema agisce sia da termostato sia da rilevatore. Ogni flacone contiene una sostanza chimica che permette di rilevare l'aumento di $\mathrm{CO}_{2}$ prodotto dalla crescita di microrganismi. Lo strumento controlla ogni dieci minuti l'aumento della fluorescenza, che è direttamente proporzionale all'aumento di $\mathrm{CO}_{2}$ presente. Una lettura positiva indica la presunta presenza di microrganismi vivi nel flacone.

Le resine presenti nei flaconi sono utili per neutralizzare in modo efficace diversi tipi di antibiotici.

\section{Ceppoteca utilizzata}

Nel presente studio sono stati analizzati 70 campioni di emocolture, di cui 35 risultati positivi per batteri Gram negativi e i rimanenti 35 per Gram positivi (identificati precedentemente con il sistema VITEK 2, bioMérieux, Francia). I germi isolati appartenevano alle seguenti specie: Pseudomonas aeruginosa, Enterobacter aerogenes, Escherichia coli, Klebsiella pneumoniae subsp. pneumoniae (Gram negativi), Staphylococcus aureus, Staphylococcus hominis, Staphylococcus epidermidis, Enterococcus faecalis (Gram positivi).

Come controlli della metodica sono stati utilizzati ceppi ATCC per Gram positivi e Gram negativi, rispettivamente Staphylococcus aureus ATCC 33862 ed Escherichia coli ATCC 25922.

\section{Antibiotici testati}

Per i Gram positivi sono stati testati i seguenti antibiotici (concentrazione finale): ciprofloxacina $(5 \mu \mathrm{g} / \mathrm{ml})$, levofloxacina (5 $\mu \mathrm{g} / \mathrm{ml})$, oxacillina $(1 \mu \mathrm{g} / \mathrm{ml})$, penicillina (10 UI/ml), vancomicina $(30 \mu \mathrm{g} / \mathrm{ml})$, linezolid $(30 \mu \mathrm{g} / \mathrm{ml})$; mentre, per i gram negativi: ciprofloxacina $(5 \mu \mathrm{g} / \mathrm{ml})$, levofloxacina $(5 \mu \mathrm{g} / \mathrm{ml})$, amikacina (30 $\mu \mathrm{g} / \mathrm{ml})$, ceftazidime $(30 \mu \mathrm{g} / \mathrm{ml})$, piperacillina $(100 \mu \mathrm{g} / \mathrm{ml})$.

\section{Antibiogramma con metodica di diffusione su piastra}

L'antibiogramma classico è stato eseg uito utilizzando dischetti antibiotati (OXOID, Regno Unito, Basingstoke) in diffusione su piastra, secondo il metodo di Kirby-Bauer consigliato da EUCAST (http://www.eucast.org/eucast_disk_diffusion_test/). Per questo test è stato utilizzato il terreno Mueller Hinton (OXOID, Regno Unito, Basingstoke) sul quale sono state seminate sospensioni batteriche della torbidità corrispondente allo 0.5 McFarland, ovvero circa $1.5 \times 10^{6}$ batteri $/ \mathrm{mL}$. Successivamente alla deposizione dei dischetti sul terreno, è stata effettuata un'incubazione a $37^{\circ} \mathrm{C}$ per 24 ore, dopo la quale, si è proceduto alla misura della larghezza degli aloni di inibizione.

\section{Antibiogramma con HB\&L}

L'HB\&L (ALIFAX, Italia) è un sistema laser nefelometrico per lo studio colturale di liquidi biologici. La soglia di sensibilità dello strumento può essere programmata da $1 \mathrm{a}$ $>10^{6}$ batteri/mL. Campioni fortemente positivi sono rilevabili anche solo dopo 45 minuti dall'inizio dell'analisi. Oltre alla determinazione del Potere Antimicrobico Residuo (rilevazione di un pregresso trattamento antibiotico), lo strumento è in grado di ottenere l'antibiogramma specifico per l'agente infettivo desiderato. Quest'ultimo aspetto risulta di particolare interesse, in quanto evita l'utilizzo di antibiotici ad ampio spettro ed il derivante incremento di resistenze a questi farmaci.

Eseguita la colorazione di Gram, direttamente da emocoltura, si è proceduto alla determinazione dell'antibiogramma con lo strumento HB\&L, che utilizza la nefelometria a raggio laser per la misurazione della cinetica di crescita batterica. Brevemente, un raggio laser polarizzato passa attraverso le provette contenenti brodo eugonico in cui è dispensato il campione. I segnali che nascono dalla diffusione della luce vengono analizzati ed elaborati da un software in modo da essere tradotti in curve di crescita che permettono di rilevare la carica microbica.

Lo strumento impiega complessivamente circa quattro ore per eseguire l'antibiogramma.

In questo studio, su emocolture positive, le sensibilità dei batteri agli antibiotici, valutate con sistema HB\&L, sono state confrontate con il metodo Kirby-Bauer (diffusione su piastra). Il confronto è stato esteso anche al sistema VITEK 2, già precedentemente utilizzato per l'identificazione dei batteri della ceppoteca impiegata nello studio.

Le emocolture sono state sottoposte a un breve protocollo di preparazione del campione, prima di essere analizzate dall'HB\&L. Brevemente, $5 \mathrm{~mL}$ di sangue dell'emocoltura sono stati centrifugati per $1 \mathrm{~min}$ a $3000 \mathrm{rpm}$, allo scopo di far sedimentare i globuli rossi. In considerazione del mezzo liquido delle emocolture e della presenza residua di emazie, anche dopo centrifugazione, la metodica utilizzata per la determinazione della torbidità dello 0.5 McFarland $\left(1.5 \times 10^{8}\right.$ batteri $\left./ \mathrm{mL}\right)$ è legata alla crescita batterica. Si è proceduto, pertanto, ad inoculare il brodo eugonico, a seconda dei casi, con quantità crescenti di centrifugato(di norma da $50 \mu \mathrm{L}$ a $100 \mu \mathrm{L}$ ), per ottenere lo 0.5 McFarland. La stessa quantità utilizzata per ottenere 0.5 Mc Farland è stata inoculata nei brodi per la determinazione dell'antibiogramma e nel brodo di controllo. Sono poi stati aggiunti $50 \mu \mathrm{g} / \mathrm{mL}$ di antibiotico, precedentemente preparato, nei rispettivi brodi e l'analisi è poi proseguita sullo strumento con incubazione variabile da 4 a 6 ore.

\section{Antibiogramma con VITEK 2}

Per l'identificazione al VITEK 2 (bioMérieux, Francia) dei batteri utilizzati nel presente studio, sono state utilizzate le card GN e GP, rispettivamente per i Gram negativi e per i Gram positivi. Per la determinazione dell'antibiogramma automatico, sempre con VITEK 2, sono invece state utilizzate le card AST-089 per i Gram negativi, AST-586 per gli streptococchi e AST-580 per gli stafilococchi, secondo quanto indicato dai protocolli consigliati dalla bioMérieux.

\section{RISULTATI e DISCUSSIONE}

Dopo una fase preliminare di calibrazione dello strumento e di ottimizzazione del protocollo analitico, per aver dati riproducibili ed eliminare le possibili interferenze date dai globuli rossi, sono stati ottenuti i dati evidenziati dalle seguenti figure e tabelle.

Nelle seguenti Figure I e II sono riportati i risultati cumulativi dei profili RIS (Resistente/Intermedio/Sensibile) ottenuti con sistema HB\&L dei batteri analizzati, sia Gram positivi, che Gram negativi, rispetto agli altri due metodi utilizzati. I risultati ottenuti sui campioni sono espressi in valori assoluti.

Nella Figura III sono, invece, evidenziati due esempi dei risultati relativi all'analisi in parallelo fra la metodica di diffusione su piastra e quella con HB\&L per Klebsiella pneumoniae spp pneumoniae e per Staphylococcus aureus.

Nella seguente Tabella 1, infine, sono riportati i valori di correlazione fra il metodo HB\&L e gli altri due descritti nei materiali e metodi.

I dati descritti nella Tabella 1 sono stati ottenuti utilizzando l'equazione relativa al calcolo del coefficiente di correlazione di Pearson. I dati evidenziano che esiste un'ottima correlazione fra i metodi manuali ed automatizzati rispetto a quelli ottenuti con il sistema HB\&L (media di 0.99, sia per i Gram positivi, che per i Gram negativi).

\section{CONCLUSIONI}

L’applicazione del sistema HB\&L per la determinazione rapi- 
da dell'antibiogramma, eseguito direttamente su emocoltura positiva, risulta molto utile per fornire al clinico in tempi idonei, le indicazioni sulla terapia antibiotica da somministrare al paziente. Il sistema è completamente automatizzato ed ha un'elevata efficienza nella rilevazione di crescita batterica e di conseguenza nella determinazione della resistenza agli antibiotici.

I tempi di refertazione (4-5 ore), di gran lunga inferiori ai metodi tradizionali, permettono di ottenere prestazioni incisive in particolare sulle sepsi. In tal modo, è possibile ottenere risultati rapidi, risparmiando almeno 24 ore (tempo necessario per l'esecuzione dei metodi tradizionali) e fornendo ai clinici i dati di antibiotico-resistenza in minor tempo.

I flaconi dell'HB\&L contengono un mezzo colturale di arricchimento adatto all'utilizzo della metodica per varie tipologie di matrici, anche diverse dalle urine.

La metodica proposta è caratterizzata dall'esecuzione diretta dell'antibiogramma da surnatante di emocoltura, ottenuto dopo centrifugazione.

I risultati ottenuti, inoltre, dimostrano che i flaconi utilizzati per le emocolture non contengono inibenti per l'esecuzione dell'antibiogramma.

La correlazione compresa nell'intervallo 0.94-0.99, rispetto al metodo di determinazione dell'antibiogramma eseguito in

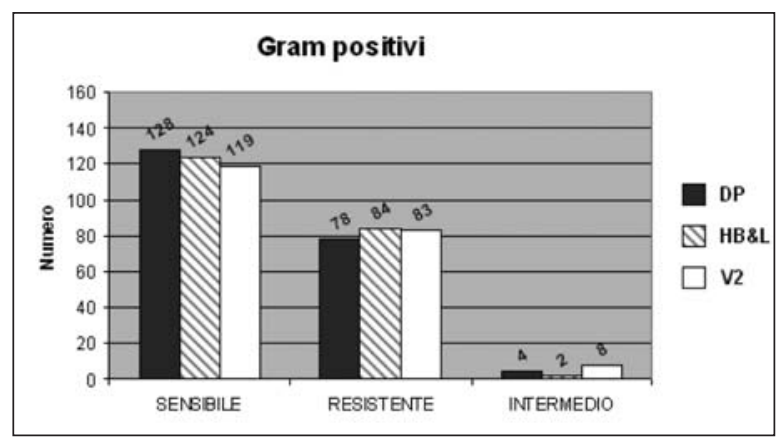

Figura I. Riepilogo dati antibiotico-resistenza per Gram positivi. DP: metodo della diffusione su piastra; HB\&L: metodo HB\&L;V2: metodo VITEK 2. Sulle ordinate è riportato il numero totale di antibiotici testati per ognuno dei $35 \mathrm{Gram}$ positivi saggiati. routine, fa dell'HB\&L uno strumento potenzialmente utilizzabile per tutti i campioni di emocolture positive provenienti dall'attività routinaria.

Tabella I: Correlazioni fra i metodi utilizzati. I dati sono stati calcolati secondo l'equazione relativa al calcolo del coefficiente di correlazione di Pearson.

\begin{tabular}{lcc}
\hline Antibiotico & $\begin{array}{c}\text { HB\&L vs } \\
\text { DIFF. PIASTRA }\end{array}$ & $\begin{array}{c}\text { HB\&L vs } \\
\text { VITEK 2 }\end{array}$ \\
\hline GRAM POSITIVI & & \\
\hline Ciprofloxacina & 0.94 & 0.99 \\
\hline Levofloxacina & 0.98 & 0.99 \\
\hline Linezolid & $\mathrm{I}$ & 0.99 \\
\hline Oxacillina & 0.99 & 0.99 \\
\hline Penicillina & $\mathrm{I}$ & 0.99 \\
\hline Vancomicina & $\mathrm{I}$ & $\mathrm{I}$ \\
\hline TOTALE GRAM POSITIVI & 0.99 & 0.99 \\
\hline GRAM NEGATIVI & & \\
\hline Amikacina & 0.96 & 0.94 \\
\hline Ciprofloxacina & 0.99 & $\mathrm{I}$ \\
\hline Levofloxacina & 0.99 & $\mathrm{I}$ \\
\hline Ceftazidime & $0.8 \mathrm{I}$ & 0.96 \\
\hline Piperacillina & 0.98 & $\mathrm{I}$ \\
\hline TOTALE GRAM NEGATIVI & 0.99 & 0.99 \\
\hline
\end{tabular}

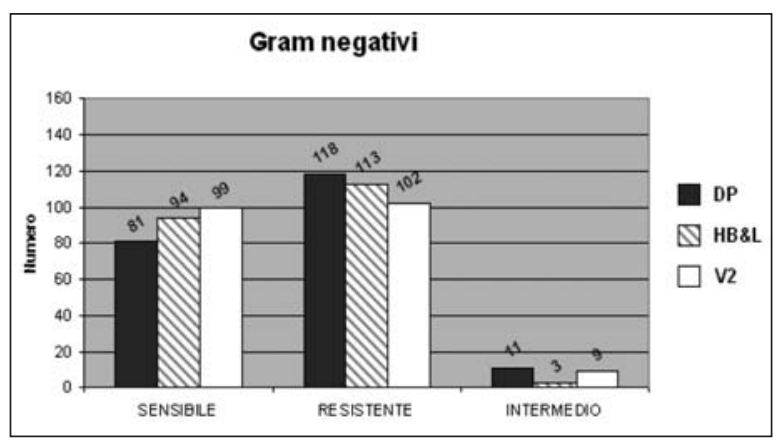

Figura II. Riepilogo dati antibiotico-resistenza per Gram negativi. DP: metodo della diffusione su piastra; $H B \& L$ : metodo $H B \& L ; V 2$ : metodo VITEK 2. Sulle ordinate è riportato il numero totale di antibiotici testati per ognuno dei 35 Gram negativi saggiati.
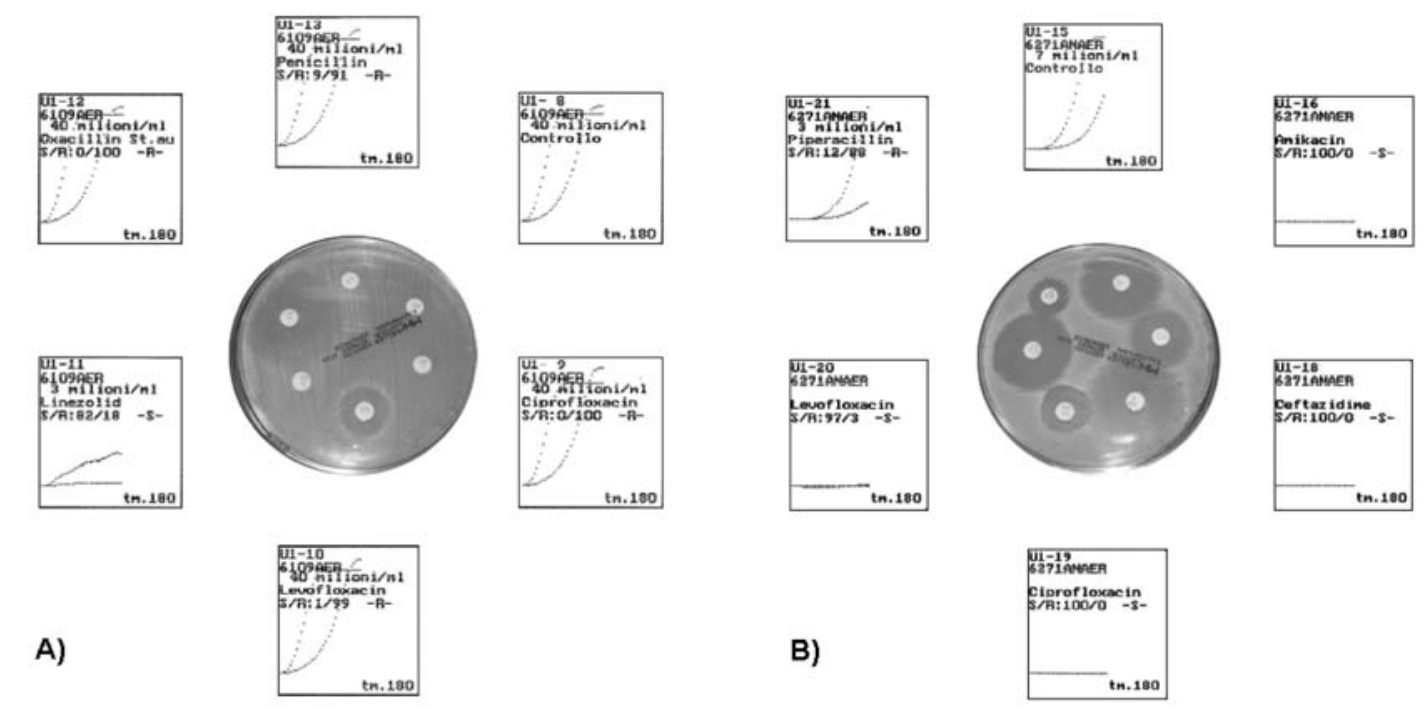

Figura III. Correlazione fra metodica di diffusione su piastra e HB\&L. Risultati ottenuti per A) Klebsiella pneumoniae spp pneumoniae e per B) Staphylococcus aureus. Nei grafici le curve di crescita evidenziano la resistenza batterica verso lo specifico antibiotico, in relazione al comportamento fenotipico col metodo della diffusione su piastra. 


\section{RINGRAZIAMENTI}

Un ringraziamento particolare alla Dott.ssa Anna Maria Grossi (ALIFAX, Italia), che ha provveduto alla collaborazione nella messa a punto della metodica.

\section{BIBLIOGRAFIA}

1. Diekema DJ, Lee K, Raney P, Herwaldt LA, Doern GV, Tenover FC. Accuracy and appropriateness of antimicrobial susceptibility test reporting for bacteria isolated from blood cultures. J Clin Microbiol. 2004 May;42(5):2258-60.

2. Fontana C, Favaro M, Minelli S, Bossa MC, Altieri A, Favalli C. A novel culturing system for fluid samples. Med Sci Monit. 2009 Feb;15(2):BR55-60.

3. Jorgensen JH, Ferraro MJ. Antimicrobial susceptibility testing: a review of general principles and contemporary practices. Clin Infect Dis. 2009 Dec 1;49(11):1749-55. Review.

4. McGill K, Kelly L, Madden RH, Moran L, Carroll C, O’Leary A, Moore JE, McNamara E, O’Mahony M, Fanning S, Whyte P. Comparison of disc diffusion and epsilometer (E-test) testing techniques to determine antimicrobial susceptibility of Campylobacter isolates of food and human clini- cal origin. J Microbiol Methods. 2009 Nov;79(2):238-41.

5. Mittman SA, Huard RC, Della-Latta P, Whittier S. Comparison of BD phoenix to vitek 2, microscan MICroSTREP, and Etest for antimicrobial susceptibility testing of Streptococcus pneumoniae. J Clin Microbiol. 2009 Nov;47(11):3557-61

6. Mokaddas EM, Salako NO, Philip L, Rotimi VO. Discrepancy in antimicrobial susceptibility test results obtained for oral streptococci with the Etest and agar dilution. J Clin Microbiol. 2007 Jul;45(7):2162-5.

7. Pikkemaat MG, Rapallini ML, Dijk SO, Elferink JW. Comparison of three microbial screening methods for antibiotics using routine monitoring samples. Anal Chim Acta. 2009 Apr 1;637(1-2):298-304.

8. Roveta S, Marchese A, Debbia EA. Evaluation of the Uro-Quick, a new rapid automated system, for the detection of well-characterized antibioticresistant bacteria. J Chemother. 2004 Apr;16(2):107-18.

9. Roveta S, Marchese A, Debbia EA. Antibiotic susceptibility tests directly on urine samples using Uro-Quick, a rapid automated system. J Chemother. 2006 Feb;18(1):12-9.

10. Stone ND, O'Hara CM, Williams PP, McGowan JE Jr, Tenover FC. Comparison of disk diffusion, VITEK 2, and broth microdilution antimicrobial susceptibility test results for unusual species of Enterobacteriaceae. J Clin Microbiol. 2007 Feb;45(2):340-6. 